\title{
Predictores de progresión de enfermedad renal en el paciente anciano
}

\author{
Manuel Heras Benitoํㅜ María José Fernández Reyes Luis ${ }^{2}$ \\ ${ }^{1}$ Servicio de Nefrología. Complejo Asistencial Universitario de Salamanca. España \\ ${ }^{2}$ Servicio de Nefrología. Hospital General de Segovia. España
}

\section{Resumen}

El riñón del anciano experimenta cambios estructurales y funcionales, que lo convierte en más vulnerable para la enfermedad renal. La mayoría de ancianos con enfermedad renal crónica fallece antes de desarrollar progresión renal. Sin embargo, el principal grupo de pacientes incidentes de nefropatía terminal es el de personas mayores de 65 años, representando la diabetes mellitus y las causas vasculares sus principales etiologías. En esta revisión se analizan elementos renales (filtrado glomerular, proteinuria/albuminuria, potasio, fracaso renal agudo y diuréticos) y factores geriátricos (estado funcional, cognitivo y fragilidad) como predictores de progresión de enfermedad renal. La identificación de estos factores contribuirá a establecer estrategias de prevención renal y al pronóstico de la enfermedad en la toma decisiones.

PALABRAS CLAVE: anciano; enfermedad renal crónica; fracaso renal agudo; progresión renal; ecuaciones de predicción; fragilidad.

\section{Predictors of progression of renal disease in the elderly patient}

\section{Summary}

The kidney in elderly people suffers structural and functional changes, which makes it more vulnerable

\section{Correspondencia:}

Manuel Heras Benito

Servicio de Nefrología. Complejo Asistencial U. de Salamanca

Paseo de San Vicente n 58. 37007 Salamanca

E-mail: mherasb@saludcastillayleon.es to kidney disease. The majority of the elderly with chronic kidney disease die before developing renal progression. However, the main group of patients that develop terminal nephropathy is that of people older than 65 years, representing diabetes mellitus and vascular causes the main etiologies. In this review, renal elements (glomerular filtration, proteinuria/ albuminuria, potassium, acute renal failure and diuretics) and geriatric factors (functional, cognitive and frail state) are analyzed as predictors of renal disease progression. The identification of these factors will help to establish strategies for renal prevention and the prognosis of the disease in decision making.

KEYWORDS: elderly; chronic kidney disease; acute renal failure; renal progression; prediction's equations; frailty.

\section{Introducción}

El envejecimiento "normal" se acompaña de cambios en el riñón, que afectan tanto a su morfología como al funcionamiento renal, que hacen que este órgano, per se, sea especialmente vulnerable para desarrollar enfermedad renal ${ }^{1}$. Además, en la población anciana, es común la presencia de multimorbilidad (diabetes mellitus, hipertensión y/o síndromes geriátricos), que puede condicionar el pronóstico de la enfermedad renal ${ }^{2,3}$. También, la edad por sí misma, influye en el pronóstico renal: 0’Hare describió el efecto modulador de la edad sobre la enfermedad renal, tras realizar un seguimiento de más de 3 años, en más de 200.000 veteranos americanos, con enfermedad renal crónica (ERC) estadios 3-5: para un mismo nivel de filtrado glomerular ( $F G$ ), a medida que se incrementaba la edad, el pronóstico "mortalidad" superaba al de progresión de ERC a nefropatía terminal. En personas mayores de 85 años, esta afirmación era independiente del grado de $\mathrm{FG}$ basal que presentaran al inicio del estudio ${ }^{4}$. Diversos estudios han puesto de manifiesto estos hallazgos, de elevada mortalidad frente al número 
de pacientes con ERC que llegan a un estadio de nefropatía terminal ${ }^{4-6}$. Sin embargo, a pesar de esta menor proporción de ancianos con ERC que llegan a nefropatía terminal, en el informe del registro de diálisis y trasplante de la Sociedad Española de Nefrología del año 2016, se pone de manifiesto que es el grupo de pacientes mayores de 65 años, donde se alcanza la mayor incidencia de nefropatía terminal, con un pico máximo de 455 casos por millón de población en personas de $>75$ años $^{7}$. $Y$ respecto a sus causas, la diabetes mellitus y las causas vasculares representan las principales etiologías en el grupo de 6575 años y en $>75$ años respectivamente ${ }^{7}$. Respecto a la evolución de la función renal, en el estudio longitudinal de Baltimore, con un seguimiento de veinte años de 254 voluntarios sanos, se demostró un descenso en el aclaramiento de creatinina por promedio de $0,75 \mathrm{ml} / \mathrm{min} / \mathrm{año}^{8}$. En el estudio de Hemmeralgan et al, con más de 10.000 personas de 66 años o más, y dos años de seguimiento, se comprobó que la mayoría de pacientes, sobre todo, si no tenían proteinuria, no presentaban progresión renal o era mínima; los pacientes con diabetes mellitus o FG menor a $30 \mathrm{ml} / \mathrm{min}$ en el reclutamiento, eran los que presentaban mayor progresión renal ${ }^{9}$. Sin embargo, existen determinados factores que pueden afectar a la trayectoria de función renal y, por tanto, condicionar el pronóstico $0^{10,11}$. En esta revisión se analizan elementos propiamente "renales" y determinados aspectos "geriátricos" asociados con la progresión de la enfermedad renal en ancianos.

\section{Principales causas de enfermedad renal crónica que condicionan nefropatía terminal}

La diabetes mellitus y la hipertensión arterial son factores de riesgo para el desarrollo de ERC ${ }^{12}$. Y ambas patologías suelen incrementar su prevalencia en la población anciana: la disminución en la secreción de insulina junto con déficits mitocondriales, así como cambios en la expresión de genes que regulan la elasticidad e hipertensión arterial, hacen que tanto la diabetes mellitus como la hipertensión arterial, sean morbilidades frecuentes durante el proceso de envejecimiento ${ }^{12}$. En un trabajo reciente español, en el que se analizó la prevalencia de ERC en personas con diabetes mellitus y edad $>64$ años, ésta se situó en un $37,2 \%$, y la presencia de insuficiencia renal (definida por filtrado glomerular menor a $60 \mathrm{ml} / \mathrm{min} / 1,73$ $\mathrm{m}^{2}$ ) fue del $29,7 \%{ }^{13}$. En este estudio el deterioro renal se relacionó con la duración de la diabetes, mayor edad, peor control glucémico, enfermedad micro y macrovascular así como mayor número de hipoglucemias, dependencia y comorbilidad $^{13}$. Sin embargo, en otras series esta prevalencia puede ser aún mayor, como en el estudio de Lamine
F et al, con una prevalencia de ERC del $45 \%{ }^{14}$. E incluso cercana al $60 \%$, como ocurre en el trabajo reportado por Kim et $\mathrm{al}^{15}$. En este último estudio también se refleja que la mayor tasa de progresión renal se relacionaba con una duración mayor de 10 años de la diabetes mellitus ${ }^{15}$. Las causas vasculares representan el $48,2 \%$ de la incidencia de nefropatía terminal en personas mayores de 75 años $^{7}$. Dentro de las causas vasculares, la hipertensión arterial es un factor de riesgo conocido para el desarrollo de la enfermedad cardiovascular y de la ERC ${ }^{12,16}$. Con el envejecimiento poblacional, la prevalencia de hipertensión arterial se incrementa, pudiendo ser superior al 50\%, y en aproximadamente la mitad de los casos se trata de una hipertensión sistólica aislada ${ }^{16}$. En algunos estudios específicos en personas mayores de 80 años (estudio Hypertension in the Very Elderly Trial, HYVET) ${ }^{17}$, 0 en el estudio (Systolic Blood Pressure Intervention Trial, SPRINT ${ }^{18}$, mostraron los beneficios de reducción de las cifras de presión arterial sobre eventos cardiovasculares y la mortalidad. Sin embargo, estos estudios presentan la limitación de que la población incluida era relativamente sana, con inclusión de pocos pacientes frágiles y con mala situación funcional19. En estos últimos pacientes, el control estricto de presión arterial (con mismos objetivos de control de presión que para población con buena situación funcional) debería de reconsiderarse para evitar efectos secundarios, como deterioro renal agudo de origen hemodinámico (hipoperfusión renal), que en algunos casos puede ser irreversible ${ }^{19}$.

\section{Elementos renales}

Filtrado glomerular: fórmulas de estimación y la formula hematocrito, urea y género (HUGE).

EI FG reducido (menor reserva renal) también se ha asociado con mayor tasa de progresión renal, como se describe en el estudio de Hemmelgarn et $\mathrm{al}^{8} \mathrm{y}$ con el riesgo de llegar a un estadio de nefropatía terminal ${ }^{20}$.

Aunque el FG es considerado el mejor marcador de función renal, a la hora de su interpretación hay que tener en cuenta: 1) la mayoría de fórmulas utilizadas para su estimación no han sido validadas en ancianos; 2) un mismo paciente puede ser etiquetado en diferente estadio de ERC según la ecuación empleada y 3) el FG puede disminuir durante el proceso de envejecimiento, sin implicar necesariamente, la presencia de una enfermedad renal ${ }^{21,22}$. En estas situaciones de encontrar un $F G$ reducido $\left(<60 \mathrm{ml} / \mathrm{min} / 1,73 \mathrm{~m}^{2}\right)$, puede ser de utilidad el uso de la fórmula hematocrito, urea y género (HUGE): si el valor $L$ es mayor a 0 , el paciente tiene $E R C^{23}$. En nuestro 
estudio de ancianos con ERC, se demuestra la capacidad predictora de HUGE sobre el deterioro renal en el seguimiento, al presentar mayor progresión renal, mayor número de episodios de fracaso renal agudo (FRA) y mayor mortalidad en aquellos pacientes con HUGE $>0$ en el periodo basal ${ }^{24}$.

\section{Proteinuria/Albuminuria:}

La proteinuria y/o albuminuria es el principal signo de daño renal: su presencia se ha relacionado con la progresión renal, desarrollo de nefropatía terminal y con la mortalidad. El empleo de fármacos antiproteinúricos -como los agentes bloqueadores del sistema renina angiotensina aldosterona- y un control estricto de la presión arterial pueden contribuir a modificar la trayectoria renal (disminuir la tasa de descenso del FG) al disminuir la proteinuria ${ }^{10}$.

\section{Potasio}

El riñón es el principal órgano responsable de la eliminación corporal del potasio. Sin embargo, los cambios renales asociados al envejecimiento (disminución del FG y de la función del túbulo distal, junto con menor producción de renina y de aldosterona), van a predisponer al paciente anciano a desarrollar hiperpotasemia ${ }^{25}$. Si además, el paciente tiene insuficiencia renal, diabetes mellitus o enfermedades cardiovasculares, en las cuales pueda estar indicado el empleo de fármacos beneficiosos en la protección cardiovascular o en la nefroprotección, como -bloqueadores del sistema renina angiotensina aldosterona-, el riesgo de hiperpotasemia se incrementa y por tanto incremento de la morbi-mortalidad ${ }^{25}$. Incluso existen evidencias crecientes de mantener la kaliemia en un margen más estrecho dentro del intervalo de la normalidad de 3,5-5 mmol/L25. En el estudio de Nakhoul et al, en una cohorte de más de 36.000 pacientes con FG $<60 \mathrm{ml} / \mathrm{min} / 1.73$, demostraron que las concentraciones de potasio $<4 \mathrm{mmol} / \mathrm{L}$ y $>5 \mathrm{mmol} / \mathrm{L}$, se asociaron con mortalidad, pero no con progresión de ERC a nefropatía terminal ${ }^{26}$. En nuestro estudio de ancianos con ERC de Segovia, también encontramos una asociación significativa, entre el valor de potasio sérico basal con la mortalidad a los 10 años, especialmente en ancianos con un valor de potasio sérico superior a la mediana de 4,45 $\mathrm{mmol} / \mathrm{L}^{27}$

\section{Fracaso renal agudo}

En los últimos años se está constatando un incremento en la incidencia de FRA en los ancianos, hasta el punto de considerarse la auténtica epidemia en nefrología ${ }^{28}$. Una posible explicación pueda encontrarse en la vulnerabilidad del riñón del anciano - relacionada con los cambios estructurales y funcionales-, y el añadido de la polifarmacia, empleo de fármacos nefrotóxicos y/o realización de pruebas diagnósticas o terapéuticas con contraste iodado; de hecho, existe una relación positiva entre la edad y la incidencia acumulada de FRA: a mayor edad, más incidencia de FRA; por lo que, la edad per se puede ser un elemento suficiente de no recuperación renal tras un episodio de $\mathrm{FRA}^{28}$. En un amplio estudio de Ishani et al, sobre una cohorte de más de 200.000 personas ancianas hospitalizadas, se comprobó el riesgo de desarrollar nefropatía terminal en aquellos pacientes con FRA y sobre todo, si éstos tenían antecedentes de ERC, sugiriendo que estos episodios de FRA aceleraban la progresión renal ${ }^{29}$. En nuestro estudio de ancianos con ERC de Segovia, con la limitación de un tamaño muestral menor, también comprobamos que aquellos pacientes que habían tenido algún episodio de FRA, eran los que tenían mayor descenso en el $F G$ en el seguimiento ${ }^{30}$. $E$ igualmente en un trabajo descriptivo trasversal, en el que analizábamos las características de la ERC en población específicamente nonagenaria, encontramos que de los 26 pacientes estudiados (edad media de 92 años), más de la mitad tenían registrados entre sus antecedentes haber sufrido algún evento de FRA ${ }^{31}$. En otro trabajo reciente, que analiza el pronóstico clínico del FRA desarrollado fuera del hospital, la incidencia global se sitúa en un 7,3\%, siendo de un $11 \%$ en población mayor de 75 años respecto a un 5,8\% en el grupo de edad entre 65-75 años ${ }^{32}$. En este trabajo, cuando se analiza el deterioro renal (el desarrollo de ERC) a los 6 meses del alta hospitalaria, se comprueba que, más de la mitad de los pacientes con FRA, habían desarrollado ERC, siendo significativamente menor la progresión renal en el grupo control de personas ancianas con creatinina sérica normal ${ }^{32}$. Por estos hallazgos, cada vez existen más evidencias que apuntan a la relación bidireccional entre FRA y ERC y viceversa ${ }^{33,34}$.

\section{Diuréticos}

Los diuréticos, son fármacos muy frecuentemente utilizados en personas ancianas, que además de ayudar a reducir las cifras de presión arterial, tienen el beneficio adicional de prevenir los episodios de insuficiencia cardiaca. Sin embargo, aparte de estos efectos beneficiosos, estos agentes también pueden contribuir al deterioro renal y/o alteraciones electrolíticas, sobre todo en casos de uso inadecuado (dosis elevadas, tratamiento a "piñón fijo", sin efectuar variaciones acordes con la volemia) ${ }^{35-37}$. Los diuréticos también fueron el grupo de agentes antihipertensivos más frecuentemente empleados en los pacientes de nuestro estudio de ancianos con ERC de Segovia: además comprobamos como los pacientes que recibían estos agentes, presentaron un incremento significativo de 
la creatinina sérica y por tanto, un descenso significativo del filtrado glomerular al año de seguimiento respecto a pacientes sin esta terapia ${ }^{38}$.

\section{Ecuaciones de predicción}

En la práctica clínica, en el paciente anciano con ERC se hace necesario la toma decisiones a la hora de informar sobre la ERC, las modalidades de tratamiento (conservador o terapia de reemplazo renal), y cómo informar, puesto que el pronóstico (nefropatía terminal-mortalidad) puede ser variable ${ }^{39}$. En estos casos, puede ser útil el uso de ecuaciones de predicción, tanto para el desarrollo de nefropatía terminal ${ }^{40}$ como de mortalidad ${ }^{41}$. Estas ecuaciones integran características sociodemográficas, y algunos parámetros renales como el FG o la albuminuria.

Sin embargo, en estos pacientes, por sus peculiaridades (edad, multimorbilidad...), quizás tenga más importancia pronóstica, la integración de elementos geriátricos considerados dentro de la valoración geriátrica integral.

\section{Elementos geriátricos}

Además del proceso de envejecimiento, la pérdida de la función renal puede ser el reflejo de multimorbilidad que acompaña a pacientes ancianos ${ }^{2,3}$. Bajo este punto de vista, la valoración geriátrica integral es una herramienta de ayuda, que considera, además de aspectos médicos, otros elementos de relevancia clínica a la hora de evaluar la enfermedad renal en ancianos, así como para establecer el pronóstico. En esta revisión se analiza el papel del estado funcional y mental, así como la fragilidad sobre la progresión de enfermedad renal.

\section{Ejercicio físico y estado funcional}

Cada vez está cobrando más importancia la evaluación del ejercicio físico durante el proceso del envejecimiento $^{42}$. Así, se conoce, que a medida que se incrementa la edad la actividad física tiende a disminuir y, por tanto, favorecer el sedentarismo ${ }^{43}$. Por otra parte, la actividad física también se ha considerado un marcador pronóstico sobre la evolución de la enfermedad renal ${ }^{43}$. En el estudio longitudinal de Robinson-Cohen et al, con un seguimiento de más de 3 años, se pone de manifiesto que a aquellas personas que reportaban mayor actividad física, presentan menor pérdida de función renal durante el seguimiento ${ }^{44}$.

Respecto al estado funcional y la capacidad para realizar actividades básicas o instrumentales de la vida diaria, en estudios de amplias cohortes se describe la pérdida de independencia en pacientes con ERC al poco tiempo de iniciar diálisis, junto con un aumento progresivo de la mortalidad 45,46 .

\section{Estado mental}

La situación mental puede condicionar per se, el pronóstico de la enfermedad renal en pacientes ancianos, como se ilustra en el caso comunicado por Rodríguez et al, en el que, una mujer octogenaria presentó un fracaso renal de novo en el contexto de una intoxicación por vitamina D por incomprensión terapéutica ${ }^{47}$. En efecto, diversos estudios han encontrado una asociación entre deterioro cognitivo y menor función renal ${ }^{48,49}$.

\section{Fragilidad}

La fragilidad, es un síndrome geriátrico que cada vez está cobrando mayor importancia en el paciente renal, por ser marcador de pronóstico adverso, al reflejar la reserva biológica frente a la edad cronológica ${ }^{50,51}$. En estos pacientes ancianos, la combinación de múltiples elementos que acompañan a la enfermedad renal (retención de toxinas urémicas, inflamación-estrés oxidativo, déficits hormonales, anemia etc), hacen que la fragilidad sea más frecuente que en la población sin enfermedad renal, y por tanto, con peor pronóstic $0^{50}$. Por eso se está recomendando el cribado de fragilidad en población anciana como una herramienta más de ayuda. Por otra parte, varios estudios han demostrado que los pacientes más frágiles experimentan mayor incidencia de FRA ${ }^{52,53}$.

En conclusión, los predictores de progresión renal en ancianos, podrían traducirse por vulnerabilidad renal; la identificación de estos elementos, contribuiría a establecer estrategias para retrasar o prevenir la progresión renal.

\section{Puntos clave}

1. Los cambios renales asociados al envejecimiento predisponen al paciente anciano al desarrollo de enfermedad renal crónica.

2. El pronóstico "mortalidad" en ancianos con enfermedad renal crónica supera al de progresión renal a "nefropatía terminal".

3. La mayoría de pacientes que llegan a nefropatía terminal son personas de 65 años o más, representando la diabetes mellitus y las causas vasculares las principales etiologías en esta población. 
4. Sobre un sustrato de vulnerabilidad -menor reserva renal asociada a la edad- determinados elementos renales (filtrado glomerular reducido, proteinuria) y geriátricos (estado funcional, estado mental y fragilidad) predicen progresión de la enfermedad renal.

5. La utilidad de ecuaciones de predicción, -que integran parte de estos elementos-, tanto para nefropatía terminal como de mortalidad, junto con la valoración geriátrica integral, representan herramientas de ayuda tanto para el manejo y pronóstico de la ERC en ancianos, como para establecer estrategias de prevención de progresión renal.

Recibido: 8 enero 2019

Revisado: 15 enero 2019

Modificado: 18 enero 2019

Aceptado: 25 enero 2019

\section{Bibliografía}

1. Zhou XJ, Rakheja D, Yu X, Saxena R, Varizi ND, Silva FG. The aging kidney. Kidney Int 2008;74(6): $710-20$.

2. Zebrowski P, Kulicki P. Age-related kidney problems. Wiad Lek. 2015;68(4 Pt 2):638-41.

3. Braun $F$, Brinkkötter PT. Decline in renal function in old age: part of physiological aging versus age-related disease. Z Gerontol Geriatr 2016;49(6):469-76.

4.0’Hare AM, Choi AI, Bertenthal $D$, Bacchetti $P_{\text {, }}$ Garg AX, Kaufman JS et al. Age affects outcomes in Chronic Kidney Disease. J Am Soc Nephrol 2007;18(10):2758-65.

5. Dalrymple LS, Katz R, Kestenbaum B, Shilipak MG, Sarnak MJ, Stehman-Breen $C$ et al. Chronic kidney disease and the risk of end-stage renal disease versus death. J Gen Intern Med 2011;26(4):379-85.

6. Lundström UH, Gasparini A, Belloco R, Qureshi AR, Carrero JJ, Evans M. Low renal replacement the-

rapy incidence among slowly progressing elderly chronic kidney disease patients referred to nephrology care: an observational study. BMC Nephrol 2017;18(1):59.

7. Sociedad Española de Nefrología. Organización Nacional de Trasplantes. Informe de diálisis y trasplante 2016. [Consultado 25 0ct 2018]. Disponible en: https://www.senefro.org/contents/webstructure/ InformeREER_2016_BURGOS.pdf

8. Lindeman RD, Tobin J, Shock NW. Longitudinal studies on the rate of decline in renal function with age. J Am Geriatr Soc 1985;33(4):278-85.

9. Hemmelgarn BR, Zhang J, Manns BJ, Tonelli M, Larsen $\mathrm{E}$, Ghali WA et al. Progression of kidney dysfunction in the community-dwelling elderly. Kidney Int 2006;69(12):2155-61.

10. Rosansky SJ. Renal function trajectory is more important than chronic kidney disease stage for managing patients with chronic kidney disease. Am J Nephrol. 2012;36(1):1-10.

11. 0'Hare AM, Batten A, Burrows NR, Pavkov ME, Taylor L, Gupta I et al. Trajectory of kidney function decline in the 2 years before initiation of long-term dialysis. Am J Kidney Dis 2012;59(4):513-22.

12. Anderson S, Halter JB, Hazzard WR, Himmelfarb $J$, Horne FM, Kaysen GA et al. Prediction, progression, and outcomes of chronic kidney disease in older adults. J Am Soc Nephrol 2009;20(6):1199-209.

13. Martínez Candela J, Sangrós González J, García Soidán FJ, Millaruelo Trillo JM, Díez Espino J, Bordonaba Bosque $D$ et al. Enfermedad renal crónica en España: prevalencia y factores relacionados en personas con diabetes mellitus mayores de 64 años. Nefrología 2018;38(4):401-13.

14. Lamine F, Lalubin F, Pitteloud N, Burnier M, Zanchi A. Chronic kidney disease in type 2 diabetic patients followed-up by primary care physicians in Switzerland: prevalence and prescription of antidiabetic drugs. Swiss Med Wkly 2016;146:w14282.

15. Kim K-S, Park SW, Cho YK, Kim S-K. Higher prevalence and progression rate of chronic kidney disease in elderly patients with type 2 diabetes mellitus. Diabetes Metab J 2018; 42(3):224-32. 
16. Sierra Benito C, Coca Payeras A. La hipertensión arterial en el anciano. Med Integral 2002; 40(10):425-33.

17. Bulpitt CJ, Beckett NS, Cooke J, Dumitrascu DL, Gil-Extremera B, Nachev $C$ et al. Results of the pilot study for the hypertension in the very elderly trial. J Hypertens 2003;21(12):2409-17.

18. Mezue K, Goyal A, Pressman GS, Matthew R, Horrow JC, Rangaswami J. Blood pressure variability predicts adverse events and cardiovascular outcomes in SPRINT. J Clin Hypertens (Greenwich) 2018;20(9):1247-52.

19. Rodríguez Mañas L, Castro Rodríguez M. Repensando el papel de la hipertensión arterial en la población anciana. Hipertens Riesgo Vasc. 2014;31(1):1-2.

20. Ohashi Y, Sakai K, Tanaka Y, Mizuiri S, Aikawa A. Reappraisal of proteinuria and estimated GFR to predict progression to ESRD or death for hospitalized chronic kidney patients. Ren Fail 2011;33 (1):31-9.

21. Heras M, Fernandez-Reyes MJ. Nuevas herramientas para abordar la función renal en ancianos: la ecuación Berlin initiative Study y la fórmula hematocrito, urea y género. Med Clin (Barc) 2016; 146(10):450-4.

22. Álvarez-Gregori J, Macías JF. Diferencias entre filtrado glomerular disminuido e insuficiencia renal: riesgos de asociar estos 2 conceptos en ancianos sanos. Rev Esp Ger Gerontol. 2014;49(4):184-7.

23. Alvarez-Gregori JA, Robles NR, Mena C, Ardanuy $R$, Jauregui $R$, Macías Nuñez JF. The value of a formula including haematocrit, blood urea and gender (HUGE) as a screening test for chronic renal insufficency. J Nutr Health Aging 2011;15 (6):480-4.

24. Heras M, Fernández-Reyes MJ, Guerrero MT, Muñoz A. Valor pronóstico de la fórmula HUGE en el seguimiento de la enfermedad renal en ancianos. Dial Traspl 2013;34(3):115-19.

25. Heras M, Fernandez-Reyes MJ. Concentraciones séricas de potasio: importancia de la normopotasemia. Med Clin (Barc) 2017;148:562-65.
26. Nakhoul GN, Huang H, Arrigain S, Jolly SE, Schold JD, Nally JV Jr, Navaneethan SD. Serum Potassium, End-Stage-Renal-Disease and mortality in chronic kidney disease. Am J Nephrol 2015;41(6): 456-63.

27. Heras Benito M, Fernández-Reyes MJ, Guerrero Díaz MT, Muñoz Pascual A. Niveles de potasio sérico y mortalidad a largo plazo en ancianos con hipertensión arterial. Hipert Riesgo Vasc 2017;34 (3):115-19.

28. Anderson $\mathrm{S}$, Eldadah $\mathrm{B}$, Halter JB, Hazzard WR, Himmelfarb J, McFarland Horne F et al. Acute kidney injury in older adults. J Am Soc Nephrol 2011; 22(1):28-38.

29. Ishani $A$, Xue JL, Himmelfarb J, Eggers PW, Kimmel PL, Molitoris BA et al. Acute kidney injury increases risk of ESRD among elderly. J Am Soc Nephrol 2009;20(1):223-8.

30. Heras M, Fernández-Reyes MJ, Guerrero MT, Sánchez R, Muñoz A, Molina A et al. Factores predictores de fracaso renal agudo en ancianos con enfermedad renal crónica. Nefrologia 2012;32(6):819-23.

31. Heras Benito $M$, Centeno Gómez MC, Fernández-Reyes Luis MJ, Martín Varas CR, Urzola Rodríguez G, Callejas Martínez R et al. Características clínicas de la enfermedad renal crónica (no diálisis) en nonagenarios. Nefrología 2018;38(Supl C1): S121.

32. Turgutalp K, Bardak S, Horoz M, Helvaci I, Demir S, Kiykim AA. Clinical outcomes of acute kidney injury developing outside the hospital in elderly. Int Urol Nephrol 2017;49(1):113-21.

33. Pannu N. Bidirectional relationships between acute kidney injury and chronic kidney disease. Curr Opin Nephrol Hypertens 2013; 22(3):351-6.

34. Coca SG, Cho KC, Hsu C-Y. Acute kidney injury in the elderly: predisposition of chronic kidney disease and viceversa. Nephron Clin Pract 2011;119(Supl $1)$ :c19-24.

35. Heras Benito M, Fernández-Reyes Luis MJ, Rodríguez Gómez MA. Disnatremias como consecuencia del morbus diureticus en 2 pacientes ancianos. $\mathrm{Hi}$ pert Riesgo Vasc 2014;31(1):28-30. 
36. Wehling M. Morbus diureticus in the elderly: epidemic overuse of a widely applied group of drugs. J Am Med Dir Assoc 2013;14(6):437-42.

37. Cabré M, Elias L, García M, Palomera E, Serra-Prat M. Avoidable hospitalizations due to adverse drug reactions in an acute geriatric unit. Analysis of 3292 patients. Med Clin (Barc) 2018;150(6):209-14.

38. Heras M, Fernández-Reyes MJ, Sánchez R, Guerrero MT, Prado F, Alvarez-Ude F. Repercusión sobre la función renal de los fármacos antihipertensivos utilizados en ancianos con hipertensión arterial. Hipertensión 2008;25(5):194-7.

39. Heras Benito M, Fernández-Reyes MJ. Toma de decisiones compartida en la enfermedad renal crónica avanzada del anciano. Med Clin (Barc) 2019;152(5):188-94.

40. Tangri N, Stevens LA, Griffith J, Tighiouart H, Djurdjev 0, Naimark $D$ et al. A predictive model for progression of chronic kidney disease to kidney failure. JAMA 2011;305(15):1553-59.

41. Bansal N, Katz R, De Boer IH, Peralta C.A, Fried $L F$, Siscovick DS et al. Development and validation of a model to predict 5-year risk of death without ESRD among older adults with CKD. Clin J Am Soc Nephrol 2015;10(3):363-71.

42. Sánchez E, Formiga F, Cruz-Jentoft A. La creciente importancia del rendimiento físico en la valoración geriátrica integral. Rev Esp Geriatr Gerontol 2018; 53(5):243-44.

43. Berger JR, Jaikaransingh V, Hedayati S. End-stage Kidney Disease in the elderly: approach to dialysis initiation, choosing modality, and predicting outcomes. Adv Chronic Kidney Dis 2016;23(1):36-43.

44. Robinson-Cohen C, Littman A J, Duncan GE, Weiss NS, Sachs MC, Ruzinski J et al. Physical activity and change in estimated GFR among persons with CKD. J Am Soc Nephrol 2014;25(2):399-406.
45. Kurella Tamura M, Covinsky KE, Chertow GM, Yaffe K, Landefeld CS, McCulloch CE. Functional status of elderly adults before and after initiation of dialysis. N Engl J Med 2009;361(16):1539-47.

46. Jassal SV, Chiu E, Hladunewich M. Loss of Independence in patients starting dialysis at 80 years of age or older. N Eng J Med 2009;361(16):1612-3.

47. Rodríguez $R$, Heras $M$, Fernández-Reyes MJ, Sánchez R. Fracaso renal como consecuencia de una intoxicación por vitamina $D$ en una anciana por incomprensión terapéutica. Rev Esp Geriatr Gerontol 2010;45(4):242-3.

48. Kurella Tamura M, Wadley $V$, Yaffe $K$, McClure LA, Howard G, Go R et al. Kidney function and cognitive impairment in US adults: the Reasons fog Geographic and Racial Differences in Stroke (REGARDS) Study. Am J Kidney Dis 2008;52(2):227-34.

49. Guerrero MT, Heras $M$, Muñoz $A$, Ridruejo $E$, Centeno $C$, Macias MC et al. Situación mental y funcional en ancianos con diferentes grados de filtrado glomerular. Dial Traspl. 2014;35(1):15-19.

50. Portilla Franco ME, Tornero Molina F, Gil Gregorio P. La fragilidad en el anciano con enfermedad renal crónica. Nefrologia 2016;36(6):609-15.

51. Rockwood K, Mitnitski A. Frailty in relation to the accumulation of deficits. J Gerontol A Biol Sci Med Sci. 2007;62(7):722-7.

52. Baek SH, Lee SW, Kim SW, Ahn SY, Yu MY, Kim KI et al. Frailty as a predictor of acute kidney injury in hospitalized elderly patients: a single center, retrospective cohort study. PLOS ONE 2016; 11(6): e0156444.

53. Morton $S$, Isted $A$, Avery $P$, Wang J. Is frailty a predictor of outcomes in elderly inpatients with acute kidney injury? A prospective cohort study. Am J Med 2018;131(10):1251-56.

Este artículo se distribuye bajo una Licencia Creative Commons Atribución-NoComercial 4.0 Internacional. https://creativecommons.org/licenses/by-nc/4.0/

Open Access (c) () \& 